$\mathbb{T}$ periodica polytechnica

Mechanical Engineering

$51 / 2$ (2007) 71-75

doi: 10.3311/pp.me.2007-2.05

web: http://www.pp.bme.hu/me

(c) Periodica Polytechnica 2007

RESEARCH ARTICLE

\section{Integration method for constitutive equation of von Mises elastoplasticity with linear hardening}

\author{
Attila Kossa
}

Received 2008-01-18

\begin{abstract}
This paper presents a summary of the new semi-analytical integration method presented in [10] for von Mises elastoplasticity model with combined linear isotropic-kinematic hardening within a small deformation range. Solutions for the case of constant strain rate and constant stress rate assumptions are also presented. Furthemore, it is shown how the general solution reduces to the particular cases of purely kinematic hardening, purely isotropic hardening and perfect plasticity, respectively.
\end{abstract}

\section{Keywords \\ elastoplasticity $\cdot$ combined hardening $\cdot$ exact integration}

\section{Acknowledgement}

This research has been supported by the National Development and Research Foundation, Hungary (under Contract: OTKA, T046488). This support is gratefully acknowledged.

\section{Introduction}

The most widely used plasticity model is the von Mises elastoplasticity. In terms of the hardening rule we can categorize the various cases as: perfect plasticity, kinematic hardening, isotropic hardening and combined hardening. Analytical solution for perfect plasticity was presented in [14], [11], [9]. Exact solution for linear kinematic hardening is derived in $[3,11,21,23,24]$. A semi-analytical solution is given in [20] for purely isotropic hardening. Due the complexity of the governing constitutive relations corresponding to the combined hardening case, fully analytical solution is not given in the literature. The problem was discussed in [5], [16], [17], [24] deriving some efficient approximate and nearly exact solutions. Efficient numerical integration techniques can be found in $[2-4,6,8,12,15,16]$. This paper focuses on the new semi-analytical solution of combined hardening materials presented in [10] and includes some remarks corresponding to the general solution.

The paper is organized as follows. The notation for equations will be introduced at the end of this section. Section 2 contains a brief review of the constitutive relations for von Mises elastoplasticity with combined linear isotropic-kinematic hardening. In Section 3 the solution corresponding to constant strain rate assumption is presented. In Section 4 the formulas will be derived when the loading is defined by constant stress rate.

Regarding notation, tensors are denoted by bold-face characters, the order of which is indicated in the text. The tensor product is denoted by $\otimes$, and the following symbolic operations apply: $\mathbf{a}: \mathbf{b}=\mathrm{a}_{i j} \mathrm{~b}_{i j}$, and $(\mathbf{C}: \mathbf{a})_{i j}=\mathrm{C}_{i j k l} \mathrm{a}_{k l}$, with the summation over repeated indices. The superscripts $T$ and -1 denote transpose and inverse, respectively, and the prefix tr refers to the trace. The symbol $\|\mathbf{a}\|=\sqrt{\mathbf{a}: \mathbf{a}}$ is used to denote a norm of second order tensor a. Furthermore, standard tensors are denoted by $\boldsymbol{\delta}$ for the second-order unit tensors, and by $\mathbf{I}$ for the symmetric fourth-order unit tensor. 
2 Rate-form constitutive equations of the von Mises elastoplasticity with combined linear kinematic and isotropic hardening

The well-known constitutive relations for the von Mises elastoplasticity model with combined linear isotropic-kinematic hardening are summarized here (for more details see [13], [18]). The classical additive decomposition of the total strain-rate is

$$
\dot{\boldsymbol{\varepsilon}}=\dot{\boldsymbol{\varepsilon}}^{e}+\dot{\boldsymbol{\varepsilon}}^{p},
$$

where $\dot{\boldsymbol{\varepsilon}}^{e}$ is the elastic and $\dot{\boldsymbol{\varepsilon}}^{p}$ is the plastic strain rate, respectively. The elastic behaviour is governed by the following constitutive relation:

$$
\dot{\boldsymbol{\sigma}}=\mathbf{D}^{e}: \dot{\boldsymbol{\varepsilon}}^{e},
$$

where the fourth-order elasticity tensor in linear isotropic elasticity takes the form:

$$
\mathbf{D}^{e}=2 G \mathbf{T}+K \boldsymbol{\delta} \otimes \boldsymbol{\delta}
$$

$\mathbf{T}=\mathbf{I}-\frac{1}{3} \boldsymbol{\delta} \otimes \boldsymbol{\delta}$ is the fouth-order deviatoric operator tensor, and $G$ and $K$ are the shear and bulk moduli, respectively. The von Mises yield function for combined isotropic kinematic hardening is defined by

$$
F(\boldsymbol{\xi}, R)=\|\boldsymbol{\xi}\|-R(\gamma) \leq 0,
$$

where $R(\gamma)$ represents the isotropic hardening law in terms of a scalar plastic state variable $\gamma$. The so-called reduced stress deviator is defined as

$$
\xi=s-\alpha,
$$

where $\boldsymbol{s}=\boldsymbol{\sigma}-\frac{1}{3} \operatorname{tr} \boldsymbol{\sigma} \boldsymbol{\delta}$ is the deviatoric stress, $\boldsymbol{\alpha}$ is the back stress describing the translation of the yield surface in the deviatoric stress space due to the kinematic hardening. For associative flow rule, the plastic strain rate tensor is found from the following expression:

$$
\dot{\boldsymbol{\varepsilon}}^{p}=\dot{\lambda} \frac{\partial F}{\partial \boldsymbol{\sigma}}=\dot{\lambda} \frac{\boldsymbol{\xi}}{\|\boldsymbol{\xi}\|},
$$

where the plastic loading parameter is given by

$$
\gamma=\int_{0}^{t} \dot{\lambda} d t
$$

The linear isotropic and kinematic hardening moduli can be expressed in the following forms:

$$
h_{\text {iso }}=M h, \quad h_{\text {kin }}=(1-M) h,
$$

where $h=2 H / 3$ and $H$ is the constant plastic hardening modulus. The mixed hardening parameter $M \in[0,1]$ defines the relation between the isotropic and kinematic part, respectively. $M=0$ stands for purely kinematic hardening, $M=1$ for the purely isotropic hardening. The linear isotropic hardening function is written as

$$
R=R_{0}+\gamma h_{i s o},
$$

where $R_{0}$ is a material constant related to the initial value of yield stress $R_{0}=\sqrt{2 / 3} \sigma_{y}$. The evolution law for the back stress tensor is defined by the Ziegler-Prager's model as

$$
\dot{\alpha}=h_{k i n} \dot{\boldsymbol{\varepsilon}}^{p}=h_{k i n} \dot{\lambda} \frac{\boldsymbol{\xi}}{\|\boldsymbol{\xi}\|} .
$$

The loading/unloading conditions can be expressed in KuhnTucker form as

$$
\dot{\lambda} \geq 0, \quad F(\xi, R) \leq 0, \quad \dot{\lambda} F(\xi, R)=0 .
$$

The plastic multiplier $\dot{\lambda}$ can be calculated using the plastic consitency condition $\dot{F}=0$ and the Eqs. (1) - (9):

$$
\dot{\lambda}=\frac{2 G \xi: \dot{\varepsilon}}{R(2 G+h)} .
$$

Finally the elastoplastic constitutive relations can be expressed as

$$
\dot{\boldsymbol{\sigma}}=\mathbf{D}^{e p}: \dot{\boldsymbol{\varepsilon}}, \quad \mathbf{D}^{e p}=\mathbf{D}^{e}-\frac{4 G^{2}}{R^{2}(2 G+h)} \boldsymbol{\xi} \otimes \boldsymbol{\xi},
$$

where $\mathbf{D}^{e p}$ is the so-called elastoplastic, or continuum tangent modulus tensor. The constitutive equation of elastoplasticity defined above can be separated into a deviatoric and a hydrostatic part as follows:

$$
\dot{\boldsymbol{s}}=2 G \dot{\boldsymbol{e}}-\frac{4 G^{2}}{R^{2}(2 G+h)} \xi(\xi: \dot{\boldsymbol{e}}), \quad \operatorname{tr} \dot{\boldsymbol{\sigma}}=3 K \operatorname{tr} \dot{\boldsymbol{\varepsilon}},
$$

where $\dot{\boldsymbol{e}}=\dot{\boldsymbol{\varepsilon}}-\frac{1}{3} \operatorname{tr} \dot{\boldsymbol{\varepsilon}} \boldsymbol{\delta}$ is the deviatoric strain rate. The rate of the $\boldsymbol{\alpha}$ is obtained from (10), (8) and (12) as

$$
\dot{\boldsymbol{\alpha}}=\frac{2 G(1-M) h}{R^{2}(2 G+h)}(\boldsymbol{\xi}: \dot{\boldsymbol{e}}) \boldsymbol{\xi},
$$

and the evolution law for the radius of the yield surface, combining (7), (8), (9) and (12) is given by

$$
\dot{R}=\frac{2 G M h}{R(2 G+h)}(\boldsymbol{\xi}: \dot{\boldsymbol{e}}) .
$$

From (14) and (15), the expression for $\dot{\xi}$ can be written as

$$
\dot{\boldsymbol{\xi}}=2 G \dot{\boldsymbol{e}}-\frac{2 G}{R^{2}}\left(1-\frac{M h}{2 G+h}\right)(\xi: \dot{\boldsymbol{e}}) \xi .
$$

\section{Time integration of constitutive equations with con- stant strain rate assumption}

Here we restrict our analysis to purely elastoplastic loading, i.e. when both the initial and the final state lie on the yield surface. The main goal is to determine the solution of Eqs. (14)(16) when the loading is given by constant strain rate. It is possible to define the following inner product between the strain rate tensor and the relative stress on the deviatoric plane (this technique was first proposed in [11] for perfect plasticity):

$$
\boldsymbol{\xi}: \dot{\boldsymbol{\varepsilon}} \equiv \boldsymbol{\xi}: \dot{\boldsymbol{e}}=\|\boldsymbol{\xi}\|\|\dot{\boldsymbol{e}}\| \cos \psi=R\|\dot{\boldsymbol{e}}\| \cos \psi .
$$




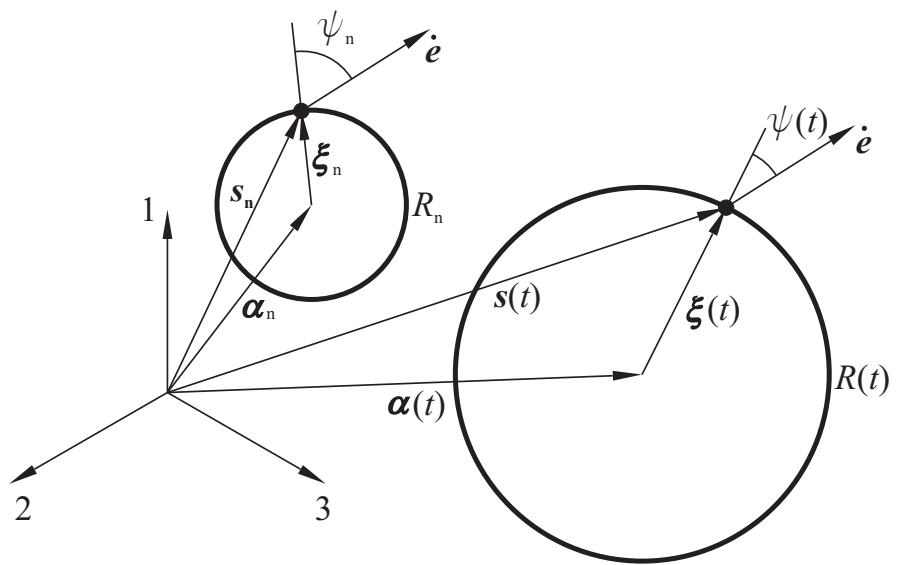

Fig. 1. Geometric interpretation of the angle $\psi$

Schematic illustration of $\psi$ is shown in Fig. 1. Using this angle variable $\psi$ after some straightforward algebraic manipulation (detailed discussion is given in [10]) we can obtain the following expression for the radius of the yield surface in terms of the angle $\psi$ :

$$
R(\psi)=R_{n}\left(\frac{\sin \psi_{n}}{\sin \psi}\right)^{a},
$$

where $\psi_{n}$ is the starting value of $\psi(t)$ computed at the known $n$-th state $(t=0)$. The dimensionless parameter $a$ is

$$
a=\frac{M c}{1+c}, \quad c=\frac{h}{2 G} .
$$

Combining Eqs. (14)-(19) finally we arrive at the following formula which implicitly defines the function $\psi(t)$ :

$\frac{4 G\|\dot{\boldsymbol{e}}\| t}{R_{n}\left(\sin \psi_{n}\right)^{a}}=B\left(\cos ^{2} \psi, \frac{1}{2},-\frac{a}{2}\right)-B\left(\cos ^{2} \psi_{n}, \frac{1}{2},-\frac{a}{2}\right)$,

where the incomplete Beta function (see [1], [19]) is defined by

$$
B(x, \nu, \mu)=\int_{0}^{x} s^{\nu-1}(1-s)^{\mu-1} d s, \quad 0 \leq x<1 .
$$

Efficient technique for computing the inverse incomplete function can be found in [7]. After $\psi(t)$ is obtained then using trigonometric identities the $\boldsymbol{\xi}(t)$ solution can be expressed as a linear combination of the relative stress $\xi_{n}$ at $n$-th state $(t=0)$ and the strain rate tensor $\dot{\boldsymbol{e}}$ as

$$
\boldsymbol{\xi}(t)=A_{\xi} \boldsymbol{\xi}_{n}+B_{\xi} \dot{\boldsymbol{e}},
$$

with the constant parameters $A_{\xi}$ and $B_{\xi}$ :

$$
A_{\xi}=\left(\frac{\sin \psi_{n}}{\sin \psi}\right)^{a-1}, \quad B_{\xi}=\frac{R_{n} \sin \left(\psi_{n}-\psi\right)}{\|\dot{\boldsymbol{e}}\| \sin \psi_{n}}\left(\frac{\sin \psi_{n}}{\sin \psi}\right)^{a} .
$$

Using (18), (19), (21) and (23) in (14) the solution for $\boldsymbol{s}(t)$ can be obtained in the following form:

$$
\boldsymbol{s}(t)=A_{s} \boldsymbol{\xi}_{n}+B_{s} \dot{\boldsymbol{e}},
$$

where the constant $A_{s}$ and $B_{s}$ are

$$
\begin{aligned}
A_{s} & =\frac{1}{(a-1)(c+1)}\left(1-A_{\xi}\right), \\
B_{s} & =\frac{2 G c}{(1+c)} t-\frac{R_{n} \cos \psi_{n}}{\|\dot{\boldsymbol{e}}\|} A_{s}+\frac{R_{n} \sin ^{a} \psi_{n}}{2(1+c)\|\dot{\boldsymbol{e}}\|} \times \\
& \left(B\left(\cos ^{2} \psi, \frac{1}{2}, 1-\frac{a}{2}\right)-B\left(\cos ^{2} \psi_{n}, \frac{1}{2}, 1-\frac{a}{2}\right)\right) .
\end{aligned}
$$

\subsection{Proportional loading}

The solution derived above is not applicable for proportional loading where $\psi_{n}=0$. When the loading is proportional then the tensor $\dot{\boldsymbol{e}}$ and $\boldsymbol{\xi}$ are coaxial, therefore we can write:

$$
\frac{\dot{\boldsymbol{e}}}{\|\dot{\boldsymbol{e}}\|}=\frac{\boldsymbol{\xi}}{R} \quad \Longrightarrow \quad \xi=R \frac{\dot{\boldsymbol{e}}}{\|\dot{\boldsymbol{e}}\|}, \quad \xi: \dot{\boldsymbol{e}}=R\|\dot{\boldsymbol{e}}\|
$$

Substituting $(28)_{2}$ and $(28)_{3}$ in $(14)_{1}$ we obtain the solution for the deviatoric stress:

$$
\dot{\boldsymbol{s}}=\frac{h}{1+c} \dot{\boldsymbol{e}} \quad \Longrightarrow \quad \boldsymbol{s}(t)=\boldsymbol{s}_{n}+\frac{h}{1+c} \dot{\boldsymbol{e}} t
$$

Combining (28) 3 and (16) the solution for the radius of the yield surface takes the form:

$$
\dot{R}=\frac{M h}{(1+c)}\|\dot{\boldsymbol{e}}\| \quad \Longrightarrow \quad R(t)=R_{n}+\frac{M h}{(1+c)}\|\dot{\boldsymbol{e}}\| t
$$

Finally the solution for the relative stress comes from $(28)_{2}$ :

$$
\boldsymbol{\xi}_{t}=\boldsymbol{\xi}_{n}+\frac{M h}{(1+c)} \dot{\boldsymbol{e}} t
$$

\subsection{Case of purely isotropic hardening}

Now the case where in Eq. (8) $M=1$, which corresponds to flow theory with purely isotropic behaviour of strain hardening is considered. In this case the centre of the yield surface is still fixed, therefore there is no back-stress $\boldsymbol{\alpha}$, i.e. $\boldsymbol{\xi} \equiv \boldsymbol{s}$. The $\psi$ angle is defined through $\boldsymbol{s}: \dot{\boldsymbol{e}}=R\|\dot{\boldsymbol{e}}\| \cos \psi$. The solution derived for combined hardening reduces to the following form:

$$
\begin{aligned}
\boldsymbol{s}(t) & =A_{s}^{i s o} \boldsymbol{s}_{n}+B_{s}^{i s o} \dot{\boldsymbol{e}}, \\
A_{s}^{i s o} & =\left(\frac{\sin \psi_{n}}{\sin \psi}\right)^{a-1}, \\
B_{s}^{i s o} & =\frac{R_{n} \sin \left(\psi_{n}-\psi\right)}{\|\dot{\boldsymbol{e}}\| \sin \psi_{n}}\left(\frac{\sin \psi_{n}}{\sin \psi}\right)^{a} .
\end{aligned}
$$

This solution with detailed calculations is presented in [20].

\subsection{Case of purely kinematic hardening}

Next the flow theory with linear kinematic hardening is discussed, in which particular case the radius of the yield surface remains unchanged and hardening occurs due only to the change of back-stress tensor. The mixed hardening parameter in this case is $M=0$. The solution (21) of $\psi(t)$ reduces to

$$
\psi(t)=2 \arctan \left(e^{\left(-\frac{2 G\|\dot{\boldsymbol{e}}\| t}{R_{0}}\right)} \tan \frac{\psi_{n}}{2}\right) .
$$


According to (23) the relative stress $\boldsymbol{\xi}(t)$ is calculated by

$$
\begin{aligned}
\boldsymbol{\xi}(t) & =A_{\xi}^{k i n} \boldsymbol{\xi}_{n}+B_{\xi}^{k i n} \dot{\boldsymbol{e}}, \\
A_{\xi}^{k i n} & =\frac{\sin \psi}{\sin \psi_{n}}, \\
B_{\xi}^{k i n} & =\frac{R_{n} \sin \left(\psi_{n}-\psi\right)}{\|\dot{\boldsymbol{e}}\| \sin \psi_{n}},
\end{aligned}
$$

where the parameters $A_{\xi}^{k i n}$ and $B_{\xi}^{k i n}$ come from (26) and (27) substituting $M=0$. The solution of the deviatoric stress $\boldsymbol{s}(t)$ is given by

$$
\begin{aligned}
\boldsymbol{s}(t) & =\boldsymbol{s}_{n}+A_{s}^{k i n} \boldsymbol{\xi}_{n}+B_{s}^{k i n} \dot{\boldsymbol{e}}, \\
A_{s}^{k i n} & =\frac{1}{(c+1)}\left(A_{\xi}^{k i n}-1\right), \\
B_{s}^{k i n} & =\frac{R_{n}}{(c+1)\|\dot{\boldsymbol{e}}\|}\left(\cos \psi-\cos \psi_{n} A_{\xi}^{k i n}\right)+\frac{2 G c}{c+1} t .
\end{aligned}
$$

\subsection{Case of perfect plasticity}

Without hardening $(h=0)$, the solution (26)-(27) reduces to the well-known form of perfect plasticity (see [11]). $\psi(t)$ can be computed by (35) with the remark that in this case the angle is defined between the deviatoric stress and the strain rate tensor. The analytical solution is represented in the form

$$
\begin{aligned}
\boldsymbol{s}(t) & =A_{s}^{p} \boldsymbol{s}_{n}+B_{s}^{p} \dot{\boldsymbol{e}}, \\
A_{s}^{p} & =\frac{\sin \psi}{\sin \psi_{n}}, \\
B_{s}^{p} & =\frac{R_{n} \sin \left(\psi_{n}-\psi\right)}{\|\dot{\boldsymbol{e}}\| \sin \psi_{n}} .
\end{aligned}
$$

\section{Time integration of constitutive equations with con- stant stress rate assumption}

In the previous section the case when the loading is given by constant strain rate was discussed. We have the so-called inverse problem when the loading path is prescribed in the stress space. Now consider the case when the stress rate assumed to be constant. The inverse of $(14)_{1}$ is written as

$$
\dot{\boldsymbol{e}}=\frac{1}{2 G} \dot{\boldsymbol{s}}+\frac{1}{R^{2} h}(\boldsymbol{\xi}: \dot{\boldsymbol{s}}) \boldsymbol{\xi} .
$$

Substituting in (17) we have

$$
\dot{\boldsymbol{\xi}}=\dot{\boldsymbol{s}}-\frac{(1-M)}{R^{2}}(\boldsymbol{\xi}: \dot{\boldsymbol{s}}) \boldsymbol{\xi} .
$$

Similarly to (18) here is also possible to define the following inner product introducing the angle variable $\omega$ :

$$
\boldsymbol{\xi}: \dot{\boldsymbol{s}}=R\|\dot{\boldsymbol{s}}\| \cos \omega
$$

Substituting (47) in (45) and using the consistency condition finally we arrive at the following solution for the radius of the yield surface (details can be found in [10]):

$$
R(\omega)=R_{n}\left(\frac{\sin \omega_{n}}{\sin \omega}\right)^{M}
$$

After quite long, otherwise straightforward manipulation the $\omega(t)$ function can be implicitly defined by the following nonlinear equation:

$\frac{2\|\dot{\boldsymbol{s}}\| t}{R_{n}\left(\sin \omega_{n}\right)^{M}}=B\left(\cos ^{2} \omega, \frac{1}{2},-\frac{M}{2}\right)-B\left(\cos ^{2} \omega_{n}, \frac{1}{2},-\frac{M}{2}\right)$.

Using trigonometric identities the relative stress $\boldsymbol{\xi}(t)$ can be written as a linear combination of $\boldsymbol{\xi}_{n}$ and the stress rate tensor $\dot{\boldsymbol{s}}$

$$
\begin{aligned}
\boldsymbol{\xi}(t) & =A_{\xi} \boldsymbol{\xi}_{n}+B_{\xi} \dot{\boldsymbol{s}}, \\
A_{\xi} & =\left(\frac{\sin \omega_{n}}{\sin \omega}\right)^{M-1}, \\
B_{\xi} & =\frac{R_{n} \sin \left(\omega_{n}-\omega\right)}{\|\dot{\boldsymbol{s}}\| \sin \omega_{n}}\left(\frac{\sin \omega_{n}}{\sin \omega}\right)^{M} .
\end{aligned}
$$

Note that the parameters $A_{\xi}$ and $B_{\xi}$ are different from thoose introduced in the previous section. The solution for the deviatoric strain takes the form:

$$
\begin{aligned}
\boldsymbol{e}(t) & =\boldsymbol{e}_{n}+A_{e} \boldsymbol{\xi}_{n}+B_{e} \dot{\boldsymbol{s}} \\
A_{e} & =\frac{1}{2 G c(M-1)}\left(A_{\xi}-1\right) \\
B_{e} & =\frac{(1+c)}{2 G c} t-\frac{R_{n} \cos \omega_{n}}{\|\dot{\boldsymbol{s}}\|} A_{e}-\frac{R_{n} \sin ^{M} \omega_{n}}{4 G c\|\dot{\boldsymbol{s}}\|} \times \\
& \left(B\left(\cos ^{2} \omega, \frac{1}{2}, 1-\frac{M}{2}\right)-B\left(\cos ^{2} \omega_{n}, \frac{1}{2}, 1-\frac{M}{2}\right)\right)
\end{aligned}
$$

\subsection{Proportional loading}

In case of proportional loading the stress rate tensor and the relative stress tensor are coaxial, i.e. we can write:

$$
\frac{\dot{\boldsymbol{s}}}{\|\dot{\boldsymbol{s}}\|}=\frac{\boldsymbol{\xi}}{R} \quad \Longrightarrow \quad \boldsymbol{\xi}=R \frac{\dot{\boldsymbol{s}}}{\|\dot{\boldsymbol{s}}\|} \quad, \quad \xi: \dot{\boldsymbol{s}}=R\|\dot{\boldsymbol{s}}\| .
$$

Substituting $(56)_{2}$ and $(56)_{3}$ in (46) gives the solution for $\boldsymbol{\xi}(t)$ as

$$
\dot{\boldsymbol{\xi}}=M \dot{\boldsymbol{s}} \quad \Longrightarrow \quad \boldsymbol{\xi}(t)=\boldsymbol{\xi}_{n}+M \dot{\boldsymbol{s}} t .
$$

The solution for $R(t)$ can be obtained by combining $(56)_{2},(56)_{3}$ and (16)

$$
\dot{R}=M\|\dot{\boldsymbol{s}}\| \quad \Longrightarrow \quad R(t)=R_{n}+M\|\dot{\boldsymbol{s}}\| t .
$$

After both $\boldsymbol{\xi}(t)$ and $R(t)$ are derived the solution of $\boldsymbol{e}(t)$ can be found by substituting Eqs. (56)-(58) in (45)

$$
\dot{\boldsymbol{e}}=\frac{1+c}{2 G c} \dot{\boldsymbol{s}} \quad \Longrightarrow \quad \boldsymbol{e}(t)=\boldsymbol{e}_{n}+\frac{1+c}{2 G c} \dot{\boldsymbol{s}} t
$$

\subsection{Case of isotropic hardening}

Solution corresponding to purely isotropic hardening is derived here. In this particular case hardening occurs only through the variation of the radius of the yield surface. Since $\boldsymbol{s} \equiv \boldsymbol{\xi}$, the angle $\omega(t)$ is defined between the deviatoric stress $\boldsymbol{s}$ and the deviatoric stress rate $\dot{\boldsymbol{s}}$ and it can be calculated from

$$
\omega(t)=\arctan \left(\frac{R_{0} \sin \omega_{n}}{\|\dot{\boldsymbol{s}}\| t+R_{0} \cos \omega_{n}}\right)
$$


which formula comes from (49) by substituting $M=1$. The solutions (53)-(55) reduce to the following expression

$$
\begin{aligned}
& \boldsymbol{e}(t)=\boldsymbol{e}_{n}+A_{e}^{i s o} \boldsymbol{s}_{n}+B_{e}^{i s o} \dot{\boldsymbol{s}} \\
& A_{e}^{i s o}=\frac{1}{2 G c} \ln \left(\frac{\sin \omega_{n}}{\sin \omega}\right) \\
& B_{e}^{i s o}=\frac{(1+c) \Delta t}{2 G c}-\frac{R_{n} \cos \omega_{n}}{\|\dot{\boldsymbol{s}}\|} A_{e}^{i s o}-\frac{R_{n} \sin \omega_{n}}{2 G c\|\dot{\boldsymbol{s}}\|}\left(\omega_{n}-\omega\right) .
\end{aligned}
$$

This solution can be found in [20].

\subsection{Case of kinematic hardening}

In purely kineamtic hardening case the mixed hardening parameter is $M=0$. Equation (49) is simplified and the relation for $\omega(t)$ is given by

$$
\omega(t)=2 \arctan \left(e^{\left.-\frac{\|\dot{\boldsymbol{s}}\| t}{2 R_{0}} \tan \left(\frac{\omega_{n}}{2}\right)\right) .}\right.
$$

The solution for the relative stress $\boldsymbol{\xi}(t)$ according to (50)-(52) becomes

$$
\begin{aligned}
\boldsymbol{\xi}(t) & =A_{\xi}^{k i n} \boldsymbol{\xi}_{n}+B_{\xi}^{k i n} \dot{\boldsymbol{s}}, \\
A_{\xi}^{k i n} & =\frac{\sin \omega}{\sin \omega_{n}}, \\
B_{\xi}^{k i n} & =\frac{R_{n} \sin \left(\omega_{n}-\omega\right)}{\|\dot{\boldsymbol{s}}\| \sin \omega_{n}} .
\end{aligned}
$$

Substituting $M=0$ in formulas (53)-(55) gives the analytical solution for the deviatoric strain as

$$
\begin{aligned}
\boldsymbol{e}(t) & =\boldsymbol{e}_{n}+A_{e}^{k i n} \boldsymbol{\xi}_{n}+B_{e}^{k i n} \dot{\boldsymbol{s}}, \\
A_{e}^{k i n} & =\frac{1-A_{\xi}^{k i n}}{2 G c}, \\
B_{e}^{k i n} & =\frac{(1+c)}{2 G c} t-\frac{R_{n} \cos \omega_{n}}{\|\dot{\boldsymbol{s}}\|} A_{e}^{k i n}- \\
& -\frac{R_{n}}{2 G c\|\dot{\boldsymbol{s}}\|}\left(\cos \omega-\cos \omega_{n}\right) .
\end{aligned}
$$

\section{Conclusion}

A brief summary of the new semi-analytical solution for von Mises plasticity with combined linear kinematic and isotropic hardening is given in this paper. The case of constant strain rate and constant stress rate loading are discussed and the solutions for each cases are derived. Furthermore it has been shown how reduces all the general solution for the following particular cases: purely kinematic hardening, purely isotropic hardening, perfect plasticity.

\section{References}

1 Abramowitz M., Stegun I.A., Handbook of Mathematical Functions. Applied Mathematics Series, Vol. 55, Dover Publications, New York, 1968.

2 Artioli E., Auricchio F., Beirãoda Veiga L., Generalized midpoint integration algorithms for $J_{2}$ plasticity with linear hardening, International Journal for Numerical Methods in Engineering 72 (2007), 422-463.
3 Auricchio F., Beirãoda Veiga L., On a new integration scheme for vonMises plasticity with linear hardening, International Journal for Numerical Methods in Engineering 56 (2003), 1375-1396.

4 Caddemi S., Computational aspects of the integration of the von Mises linear hardening constitutive laws, International Journal of Plasticity 10 (1994), 935-936.

5 Chan A.H.C., Exact stress integration for von Mises elasto-plastic model with constant hardening modulus, International Journal for Numerical and Analytical Methods in Geomechanics 20 (1996), 605-613.

6 Cocchetti G., Perego U., A rigorous bound on error in backward-difference elastoplastic time-integration, Computer Methods in Applied Mechanics and Enggineering 192 (2003), 4909-4927.

7 Dominici D., Nested derivatives: a simple method for computing series expansions of inverse functions, International Journal of Mathematics and Mathematical Sciences 2003 (2003), 3699-3715.

8 Gratacos P., Montmitonnet P., Chenot J.L., An integration scheme for Prandtl-Reuss elastoplastic constitutive equations, International Journal for Numerical Methods in Engineering 33 (1992), 943-961.

9 Hong H.K., Liu C.S., Prandtl-Reuss elastoplasticity: on-off switch and superposition formulae, International Journal of Solids and Structures 34 (1997), 4281-4304.

10 Kossa A., Szabó L., Exact intergration of the von Mises elastoplasticity model with combined linear isotropic-kinematic hardening, International Journal of Plasticity (2008), available at http://dx.doi.org/10.1016/ j.ijplas.2008.08.003.

11 Krieg R.D., Krieg D.B., Accuracies of numerical solution methods for the elastic-perfectly plastic model, Journal Pressure Vessel Technology 99 (1977), 510-515.

12 Liu C.S., A consistent numerical scheme for Mises mixed hardening constitutive equations, International Journal of Plasticity 20 (2004), 663-704.

13 Nemat-Nasser S, Plasticity. A Treatise on Finite Deformation of Heterogeneous Inelastic Materials, Dover Publications, Cambridge Univ. Press, 2004.

14 Reuss E., Berücksichtigung der elastischen Formänderung in der Plastizitätstheorie, Zeits. angew. Math. Mech. (ZAMM) 10 (1930), 266-274.

15 Ristinmaa M., Tangent modulus in Finite Element Calculations for NonLinear Materials, LUTFD2/(TFHT-3033), Div. of Solid Mechanics, Lund University (1990).

16 Ristinmaa M., Tryding J., Exact integration of constitutive equations in elasto-plasticity, International Journal for Numerical Methods in Engineering 36 (1993), 2525-2544.

17 Romashchenko V.A., Lepikhin P.P., Ivashchenko K.B., Exact solution of problems of flow theory with isotropic-kinematic hardening. Part 1. Setting the loading trajectory in the space of stress, Strength of Materials 31 (1999), 582-591.

18 Simo J.C., Hughes T.J.R., Computational Inelasticity, Springer, Berlin, 1998.

19 Spanier J., Oldham K.N., An Atlas of Functions, Springer, Berlin, 1987.

20 Szabó L., A semi-analytical integration method for J2 flow theory of plasticity with linear isotropic hardening, Computer Methods in Applied Mechanics and Engineering Submitted (2008).

21 Szabó L., Kovács Á., Numerical implemetation of Prager's kinematic hardening model in exactly integrated form for elastic-plastic analysis, Computers and Structures 26 (1987), 815-822.

22 Wei Z., Peric D., Owen D.R.J., Consistent linearization for the exact stress update of Prandtl-Reuss non-hardening elastoplastic models, International Journal for Numerical Methods in Engineering 39 (1996), 1219-1235.

23 Xucheng W., Liangming C., Exact integration of constitutive equations of kinematic hardening material and its extended applications, SMIRT-8, Brussels, Proc. Paper L2/3 (1985), 65-70.

24 Yoder P.J., Whirley R.G., On the numerical implementation of elastoplastic models, Journal Applied Mechanics 51 (1984), 283-288. 